\title{
Calculation of angular velocity, angular acceleration and torque of two common point rigid bodies using IMU
}

\author{
Dler Salih Hasan ${ }^{{ }^{*}}$, Ibrahim Hamarash ${ }^{2}$ \\ ${ }^{1}$ Department of Mechanical Engineering, Salahaddin University, Erbil, Iraq \\ ${ }^{2}$ Department of Electrical Engineering, Salahaddin University, Erbil, Iraq
}

\section{Index Terms \\ Rigid Bodies \\ Angular Acceleration \\ Torque}

Received: 22 December 2016

Accepted: 26 February 2017

Published: 30 June 2017

\begin{abstract}
This study aims to calculate the angular velocity, angular acceleration, and torque of two rigid bodies that share a common point using instantaneous sensor measurements. The goal of this approach is to produce outcome measurements free from accumulated error. IMU sensors and accelerometers have been used to capture real-time data. This arrangement applies to cases such as when the bodies are connected by a ball-and-socket joint, a Hooke joint, or a revolute joint, especially where it is impractical to use a joint measurement sensor between the bodies the relative motion of human limbs. The proposed system has been designed, built, and tested in a lab, which showed satisfactory results. The proposed sensor assembly overcomes these limitations. The case study showed accurate data capturing without any limitations. This paper has also demonstrated various formulas and algorithms that can help to calculate both robot parameters with these different sensors, which help to find out angular acceleration, torque, and angular velocity for smooth and reliable operations. The proposed circuitry system and algorithm showed very accurate results of these parameters.
\end{abstract}

\section{INTRODUCTION}

Consider two rigid bodies that share a common point $\mathrm{Q}$ as shown in Fig. 1. Such a situation will exist if the two bodies are connected by a ball-and-socket joint, a Hooke joint, or a revolute joint. A coordinate system is attached to each body and it is assumed that coordinates of point $\mathrm{P}$ in fig. 1 are known in terms of coordinate systems $A$ and $B$. In the figure, point $\mathrm{Q}$ lies on the $\mathrm{Z}$ axis of the two coordinate systems.

\footnotetext{
${ }^{*}$ Corresponding author: Dler Salih Hasan

†Email: dlear.hassan@gmail.com
}

(c) 2017 The Author(s). Published by TAF Publishing.

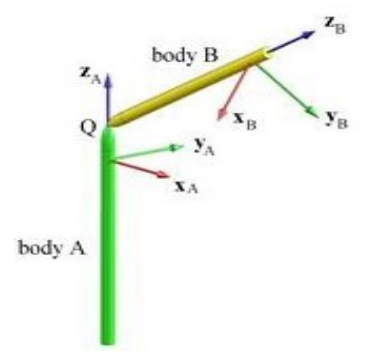

Fig. 1. Two rigid bodies that have one common point

For this approach, three 3-axis accelerometers and one 9DOF IMU (Inertia Measurement Unit) which contains (3-axis Accelerometer, 3-axis gyroscope, and 3-axis magnetometer) are attached to each body as shown in Fig. 2. The accelerometer origin points are not co-planar, no three are co-linear, and no two are coincident. Here it is assumed that the accelerometer axes are parallel to the axes of the refer- 
ence coordinate system that is attached to that body. This sensor will report the direction of the magnetic field relative to the rigid body reference coordinate system [1].

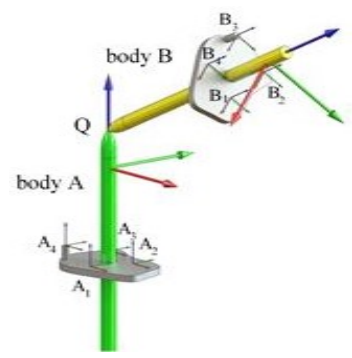

Fig. 2. Three 3-axis accelerometers and one 9DOF IMU attached to each body

The precise problem statement is given as follows:

- Two rigid bodies with reference coordinate systems A and $B$ that are connected by at least one common point.

- The coordinates of the common point $Q$ measured in both the $\mathrm{A}$ and $\mathrm{B}$ coordinate systems, i.e. APQ and BPQ.

- The locations of the sensors measured with respect to the rigid body reference coordinate system, i.e. APAi and $\mathrm{BPBi}$, $\mathrm{i}=1 . .4$.

- The accelerations of points PAi and PBi, $\mathrm{i}=1 . .4$, measured in the fixed ground coordinate system, i.e. FaAi and $\mathrm{FaBi}$, $\mathrm{i}=1 . .4$.

- The direction of the earth's magnetic field measured in the body A and B coordinate systems, i.e. ASmag and BSmag. It may be assumed that these are unit vectors [2].

- The angular velocity of body B relative to body A, i.e. A $\omega$ B. - The angular acceleration of body B relative to body A, i.e. $\mathrm{A} \omega \mathrm{B}$.

- Torque on each body.

\section{LITERATURE REVIEW}

Many scholars have used inertia sensors to track motion around the limb. Technological advancement has led to the development of effective, portable and affordable inertia sensors. As a result, a significant number of modern technologies has led to the development of wearable inertial sensors which in most cases use gyroscope, magnetometer and accelerometers [3] as the main components of tracking robot arm and human body kinematics. The major reasons to use this technology in every field are its ease of usability, light weight and smooth operation. [4] A study undertaken by [5] points out to the inherent errors due to double integration. The technique developed employed the ISSN: 2414-3103

DOI: 10.20474 /japs-3.2.3 use of direct knee angle measurement without employing the use of integration and employed the use of eight uniaxial accelerometers that were attached to the leg and thigh of the subjects.

In their analysis, they came up with a method to measure joint flexion angle in humans. To measure the knee joint angles, the researchers used two inertial measurement units comprising of IMU (gyroscopes and accelerometers) that were attached to the shank and thigh and calculated the flexion as the difference between the shank and thigh tilt angles. A gyro free orientation measurement method using low-cost accelerometer and magnetometer was proposed by [6]. To keep the system cost low, the results they got of attitude and heading were acceptable. However, [7]

corrected the gyroscope's drift in head tracking through the use of occasional measurements that were obtained from complementary Kalman filter and gravimetric tilt sensing. Other researchers such as [8] estimated the thigh and shank angle inclinations through the use of integrating gyroscopes and accelerometer data. They employed two gyroscopes and eight accelerometers and obtained an error of less than 30 for the inclination angles of shanks.

It has to be noted that the integration of inertial sensor measurements presents problems that have to do with inaccuracies. The accumulation of these inaccuracies in measurements degrades the tracking accuracy. In addition, when the integration of noisy accelerometer measurements occurs twice, it leads to a position error growing cubically over time [9], however, as postulated by [10], the use of integrating inertial sensor measurements is characterized with various limitations and inaccuracies which eventually degrade the tracking accuracy. Moreover, as noted by him, integration of noisy gyroscope eventually led to an estimated drift of 10-25oC after every minute. The system used by Nonetheless, [11] were able to design a system that uses accelerometers only.

The designed system was effective in analyzing inertia sensing using both angular acceleration and angular velocity. Nonetheless, the system that used the accelerometer, magnetometer, and gyroscopes as filtering devices, and the gyroscope-free system of accelerometers only, were faced with the challenge of integration error accumulation. The sensor calculations in the systems implemented the concept of rigid bodies and took the measurement of the orientation using an analytically simple technique that excluded the estimation criterion [12]. According to [13], Inertial Measurement Unit (IMU) is comprised of advanced tools such as ac- 
celerometers, gyroscopes and magnetometer which help to track translational and rotational movements. They cited that the devices have proved to be efficient for calculating the different movements of both the humans and robots including hip joints and wrists.

Moreover, [14] showed that gyroscopes could measure angular displacement or velocity along a single sensitive axis. Kinematic models technique using Newton-Euler equations by [15] to calculate can be applied to measure the angles of human elbow and shoulder by using wireless and wearable Inertial Measurement Units (IMU) to calculate joint angle, Angular velocity and Angular acceleration. The inverse dynamics analysis has also been a subject of study in the calculation of muscular torque [16]. With the limitations presented by other techniques, modern research focuses on understanding how inertial sensors can be important tools in the determination of muscular torque.

\section{METHODOLOGY}

The orientation relationship between coordinate systems A and B as represented by the matrix ${ }_{B}^{A} R$ [17]. Whereas linear velocity describes an attribute of a point, angular velocity describes an attribute of a body. Angular velocity describes rotational motion of a frame, if a frame is attached to the body. Whereas linear velocity describes an attribute of a point, angular velocity describes an attribute of a body. Angular velocity describes rotational motion of a frame, if a frame is attached to the body.

$A \omega B$ describes the Angular velocity of frame $\{B\}$ relative to $\{\mathrm{A}\}$.

$A \omega B$ describes the angular acceleration of frame $\{B\}$ relative to $\{\mathrm{A}\}$.

The complete algorithm for computing joint torque from the motion of the joints is composed of that link velocity and acceleration are iteratively computed from link 1 out to link $\mathrm{n}$ and the Newton-Euler equations are applied to each link. We assume that the mass distribution is extremely simple, i.e. all mass exists as a point mass at the distal end of each link. These masses are $m_{1}$ and $m_{2}$. The vectors that locate the center of mass for each link are:

${ }^{1} P_{c 1}=-l_{1} \hat{Z}_{1}$

${ }^{2} P_{c 2}=-l_{2} \hat{Z}_{2}$

Because of the point-mass assumption, the inertia tensor written at the center of mass for each link is the zero matrix:

ISSN: 2414-3103

DOI: $10.20474 /$ japs-3.2.3
${ }^{\mathrm{C} 1} l_{1}=\left[\begin{array}{lll}0 & 0 & 0 \\ 0 & 0 & 0 \\ 0 & 0 & 0\end{array}\right]$

${ }^{\mathrm{C} 2} l_{2}=\left[\begin{array}{lll}0 & 0 & 0 \\ 0 & 0 & 0 \\ 0 & 0 & 0\end{array}\right]$

There are no forces acting on the end effectors, so we have

$f_{3}=0$

$n_{3}=0$

The base of the robot is not rotating; hence, we have

$\omega_{0}=0$

$\dot{\omega}_{0}=0$

To include gravity forces, we will use

${ }^{0} \hat{v}_{0}=-g \hat{Z}_{0}$

The rotation between successive link frame is given by:

${ }_{i+1}^{i} R=R_{i}^{0 T} R_{i+1}^{0}$

${ }_{i}^{i+1} R=R_{i+1}^{0}{ }^{\mathrm{T}} R_{i}^{0}$

Assuming that angular velocity $\omega$ and angular acceleration $\hat{\omega}$. The outward iteration for link 1 is as follows, note that $\mathrm{i}=0$ :

Angular velocity

${ }^{1} \omega_{1}={ }_{0}^{1} R^{0} \omega_{0}+0{ }_{1}{ }^{1} \hat{Z}_{1}$

We have gyro IMU 1 values as ${ }^{1} \omega_{1}$ then joint angular velocity is given by:

$\dot{0}_{1}{ }^{1} \hat{Z}_{1}={ }^{1} \omega_{1}-{ }_{0}^{1} R^{0} \omega_{0}$

Angular acceleration:

$$
\begin{aligned}
& .{ }^{1} \dot{\omega}_{1}={ }_{.0}^{1} R .{ }^{0} \dot{\omega}_{0}+{ }_{.0}^{1} R .{ }^{0} \omega_{0} \times \dot{0}_{1} \cdot{ }^{1} \hat{Z}_{1}+\ddot{0}_{1} \cdot{ }^{1} \hat{Z}_{1} \\
& { }^{1} \dot{\omega}_{1} \times{ }^{1} P_{c 1}={ }^{1} \dot{v}_{c 1}-{ }^{1} \omega_{1} \times\left({ }^{1} \omega_{1} \times{ }^{1} P_{c 1}\right)-{ }^{1} \dot{v}_{c 1}
\end{aligned}
$$


If $\mathrm{A} \times \mathrm{B}=\mathrm{C}$ then $\mathrm{A}=\mathrm{B} \times \mathrm{C}$

$.{ }^{1} \dot{\omega}_{1}=.{ }^{1} P_{c 1} \times\left(.{ }^{1} \dot{v}_{c 1}-.{ }^{1} \omega_{1} \times\left(.{ }^{1} \omega_{1} \times .{ }^{1} P_{c 1}\right)-.{ }^{1} \dot{v}_{c 1}\right)$

Joint angular acceleration 1 is given by:

$\ddot{0}_{1} \cdot{ }^{1} \hat{Z}_{1}=.{ }^{1} \dot{\omega}_{1}-{ }_{.0}^{1} R \cdot{ }^{0} \dot{\omega}_{0}-{ }_{.0}^{1} R .{ }^{0} \omega_{0} \times \dot{0}_{1} \cdot{ }^{1} \hat{Z}_{1}$

Linear acceleration:

${ }^{1} \dot{v}_{c 1}={ }_{0}^{1} R\left({ }^{0} \dot{\omega}_{0} \times{ }^{0} P_{1}+{ }^{0} \omega_{0} \times\left({ }^{0} \omega_{1} \times{ }^{0} P_{1}\right)+{ }^{0} \dot{v}_{0}\right)$

Linear acceleration of the center of mass:

${ }^{1} \dot{v}_{c 1}={ }^{1} \omega_{1} \times{ }^{1} P_{c 1}+{ }^{1} \omega_{1} \times\left({ }^{1} \omega_{1} \times{ }^{1} P_{c 1}\right)+{ }^{1} \dot{v}_{1}$

We have ${ }^{0} \dot{v}_{c 1}$ (acceleration 1 measurements: $a c c_{x}, a c c_{y}, a c c_{z}$ ) solve for ${ }^{1} \dot{\omega}_{1}$.

Force:

${ }^{1} F_{1}=m_{1}{ }^{1} \dot{\omega}_{c 1}$

Momentum:

${ }^{1} N_{1}={ }^{\mathrm{C} 1} I_{1}{ }^{1} \dot{\omega}_{1}+{ }^{1} \omega_{1} \times{ }^{\mathrm{C} 1} I_{1}{ }^{1} \omega_{1}$

The outward iteration for link 2 is as follows, note that $\mathrm{i}=0$ :

Angular velocity:

${ }^{2} \omega_{2}={ }_{0}^{2} R \cdot{ }^{1} \omega_{1}+\dot{0}_{2} \cdot{ }^{2} \hat{Z}_{2}$

We have gyro IMU 1 values as ${ }^{2} \omega_{2}$ then joint angular velocity is given by:

$\dot{0}_{2}{ }^{2} \hat{Z}_{2}={ }^{2} \omega_{2}-{ }_{0}^{2} R^{1} \omega_{1}$

Angular acceleration:

$.{ }^{2} \dot{\omega}_{2}={ }_{.1}^{2} R \cdot{ }^{1} \dot{\omega}_{1}+{ }_{.1}^{2} R .{ }^{1} \omega_{1} \times \dot{0}_{2} \cdot{ }^{2} \hat{Z}_{2}+\ddot{0}_{2} \cdot{ }^{2} \hat{Z}_{2}$

${ }^{2} \dot{\omega}_{2} \times{ }^{2} P_{c 2}={ }^{2} \dot{v}_{c 2}-{ }^{2} \omega_{2} \times\left({ }^{2} \omega_{2} \times{ }^{2} P_{c 2}\right)-{ }^{2} \dot{v}_{c 2}$

If $\mathrm{A} \times \mathrm{B}=\mathrm{C}$ then $\mathrm{A}=\mathrm{B} \times \mathrm{C}$

$$
{ }^{2} \dot{\omega}_{2}={ }^{2} P_{c 2} \times\left({ }^{2} \dot{v}_{c 2}-.{ }^{2} \omega_{2} \times\left({ }^{2} \omega_{2} \times .{ }^{2} P_{c 2}\right)-.{ }^{2} \dot{v}_{c 2}\right)
$$

Joint angular acceleration 2 is given by:

$$
\ddot{0}_{2} \cdot{ }^{2} \hat{Z}_{2}=.{ }^{2} \dot{\omega}_{2}-{ }_{.1}^{2} R \cdot{ }^{1} \dot{\omega}_{1}-{ }_{.1}^{2} R \cdot{ }^{1} \omega_{1} \times \dot{0}_{2} \cdot{ }^{2} \hat{Z}_{2}
$$

Linear acceleration:

$$
{ }^{2} \dot{v}_{c 2}={ }_{1}^{2} R\left({ }^{1} \dot{\omega}_{1} \times{ }^{1} P_{2}+{ }^{1} \omega_{1} \times\left({ }^{1} \omega_{2} \times{ }^{1} P_{2}\right)+{ }^{1} \dot{v}_{1}\right)
$$

Linear acceleration of the center of mass:

${ }^{2} \dot{v}_{c 2}={ }^{2} \omega_{2} \times{ }^{2} P_{c 2}+{ }^{2} \omega_{2} \times\left({ }^{2} \omega_{2} \times{ }^{2} P_{c 2}\right)+{ }^{2} \dot{v}_{2}$

We have ${ }^{2} \dot{v}_{c 2}$ (acceleration 1 measurements: $a c c_{x}, a c c_{y}, a c c_{z}$ ) solve for ${ }^{2} \dot{\omega}_{2}$.

Force:

${ }^{2} F_{2}=m_{2}{ }^{2} \dot{\omega}_{c 2}$

Momentum:

${ }^{1} N_{2}={ }^{\mathrm{C} 2} I_{2}{ }^{2} \dot{\omega}_{2}+{ }^{2} \omega_{2} \times{ }^{\mathrm{C} 2} I_{2}{ }^{2} \omega_{2}$

The inward iteration for link 2 is as follows, note that $\mathrm{i}=0$ :

$.^{2} f_{2}={ }_{\cdot 3}^{2} R^{3} f_{3}+.^{2} f_{2}$

If $f_{3}=0$ and/or ${ }_{3}^{2} R=0$, then

${ }^{2} f_{2}={ }^{2} F_{2}$

Torque:

$.^{2} n_{2}=.^{2} N_{2}+{ }_{3}^{2} R^{3} n_{3}+{ }^{2} P_{C_{2}} \times .^{2} F_{2}+{ }^{2} P_{3} \times{ }_{3}^{2} R^{3} f_{3}$

If $n_{3}=0$ and/or ${ }_{3}^{2} R=0$, then

${ }^{2} n_{2}={ }^{2} N_{2}+{ }^{2} P_{C_{2}} \times{ }^{2} F_{2}$

For the inward iteration for link 1, the force exerted, the links are as follows, note that $\mathrm{i}=1$ :

${ }^{1} f_{1}={ }_{3}^{2} R^{2} f_{2}+{ }^{1} F_{1}$ 
Torque:

${ }^{1} n_{1}={ }^{1} N_{1}+{ }_{2}^{1} R^{2} n_{2}+{ }^{1} P_{C_{1}} \times{ }^{1} F_{1}+{ }^{1} P_{2} \times{ }_{2}^{1} R^{2} f_{2}$

Extracting the $\hat{Z}_{i}$ components of the ${ }^{1} n_{i}$, we find the joint torques:

$\tau_{i}={ }^{i} R_{i}^{T} \cdot \hat{Z}_{i}$

\section{RESULTS}

To apply the proposed model in this research paper, a wearable sensor system that consists of two links, each link with three 3-axis 345-ADXL accelerometers and one

9DOF IMU (three-axis Accelerometer, Three-axis magnetometer and three-axis gyroscope) has been designed and implemented. Fig. 3 shows the four sensors as an assembly that attach to one link. For capturing the data, a robot arm that consists of two links was designed and implemented using Arduino microcontroller as shown in Fig. 4. The system can also be mounted on the human body about human movement, geomagnetism to estimate the parameters. A firmware is developed to capture data from the sensors in both links simultaneously. As the output data from the sensors are not noise-free, Low Pass Filter is applied for accelerometer's data and Kalman filter used for 9DOF IMU data cleaning.

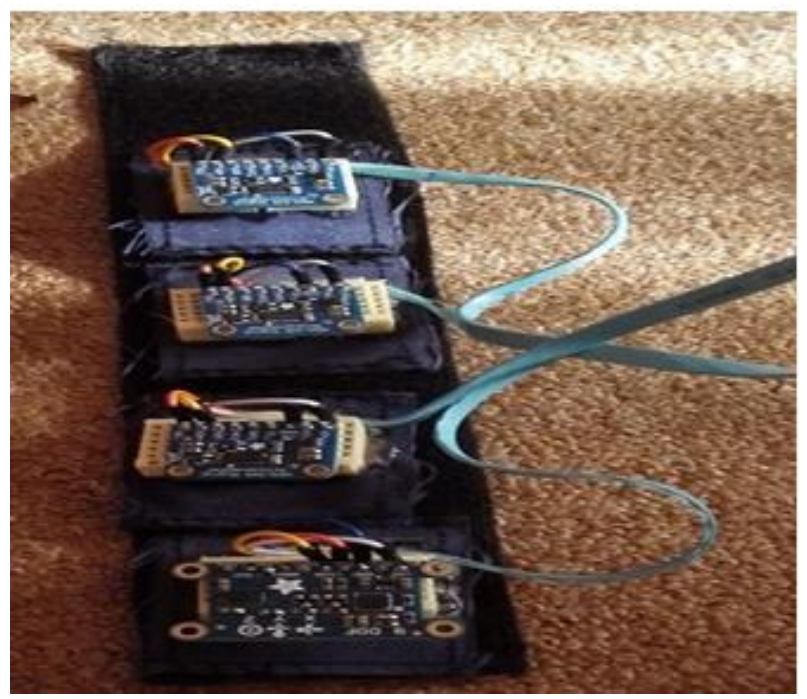

Fig. 3. Three accelerometers and One IMU attached to each link ISSN: 2414-3103

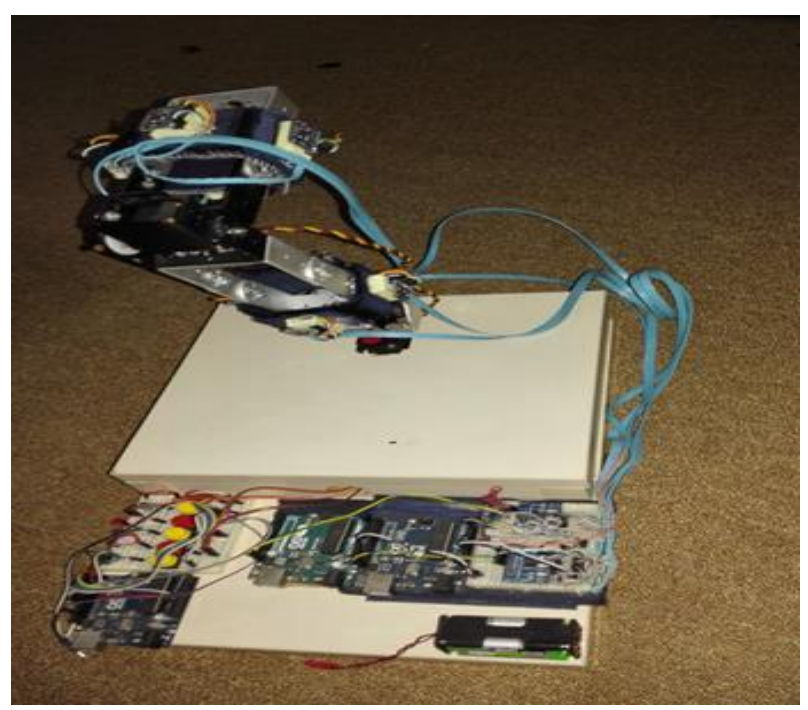

Fig. 4. Designed robot arm

An algorithm has been developed and coded using MATLAB to calculate the parameters Angular velocity, Angular Acceleration and torque in three coordinates a,y,z for both Body A and Body B which have the common point using data captured from the sensors and the model that has been developed in section 3. The Figs. 5 and 6 represent Angular velocity for body A and body B respectively, Figs. 7 and 8 represent Angular Acceleration for body A and body B respectively, while Figs. 9 and 10 represent Torque for body A and body $\mathrm{B}$ respectively.

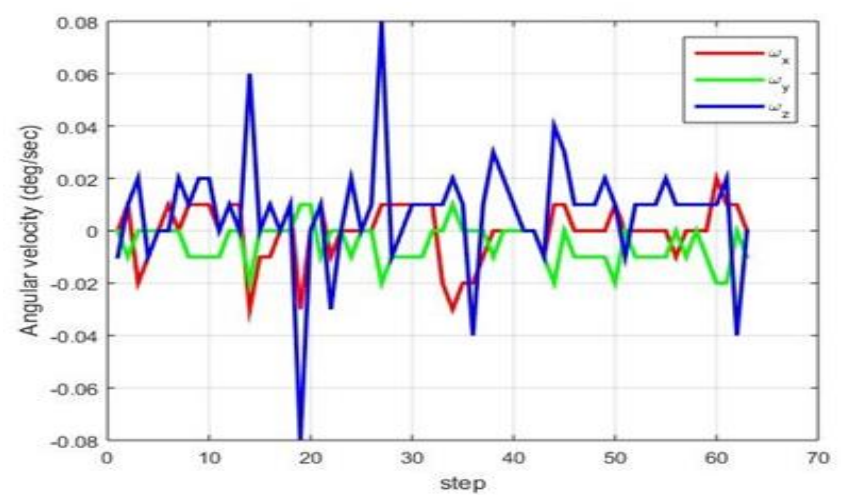

Fig. 5. Angular velocity of body A 


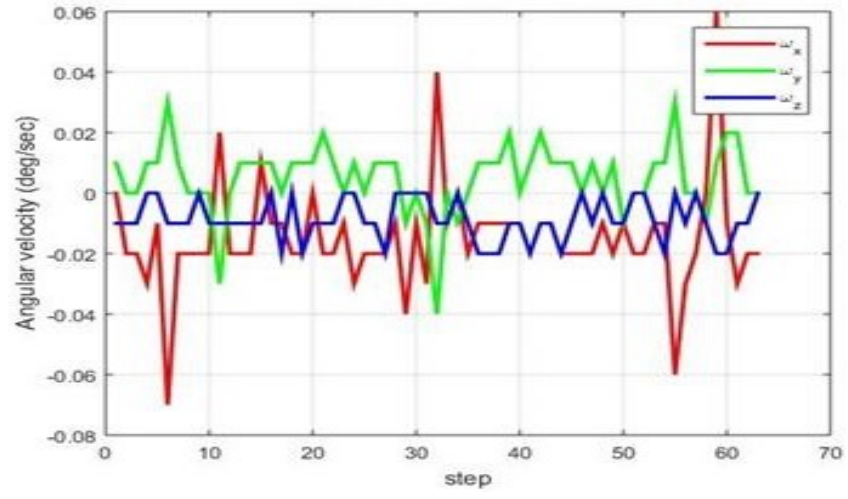

Fig. 6. Angular velocity of body B

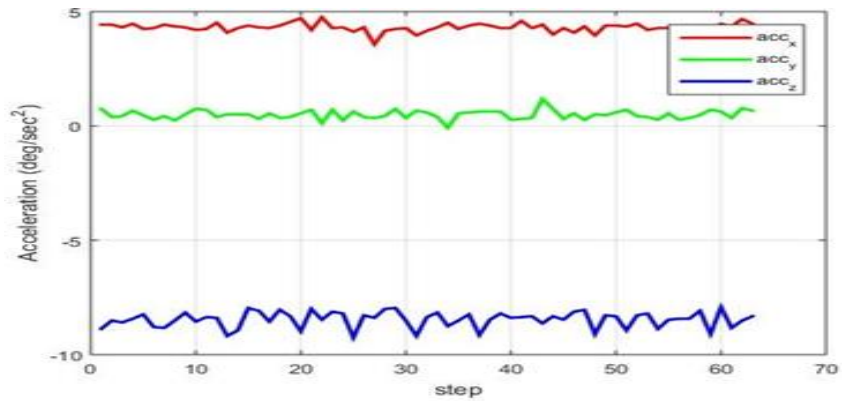

Fig. 7. Angular acceleration of body A

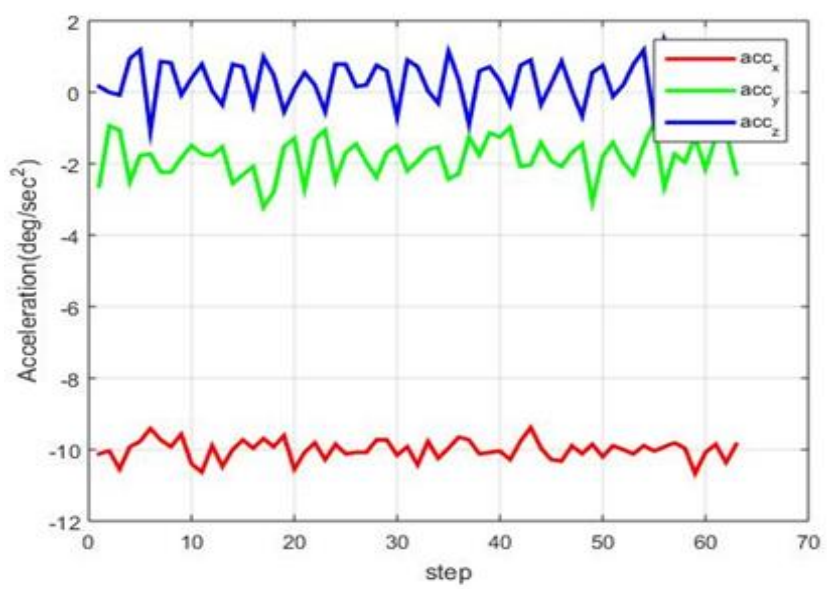

Fig. 8. Angular acceleration of body B

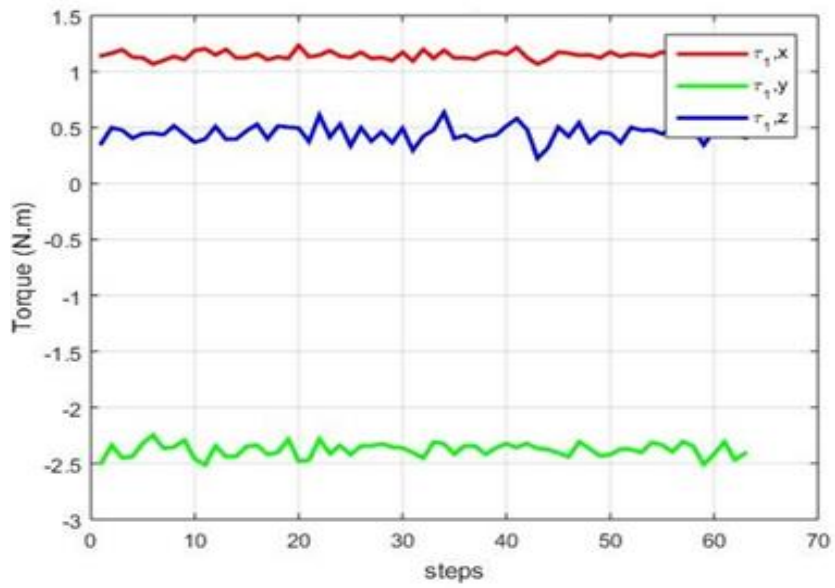

Fig. 9. Torque of body A

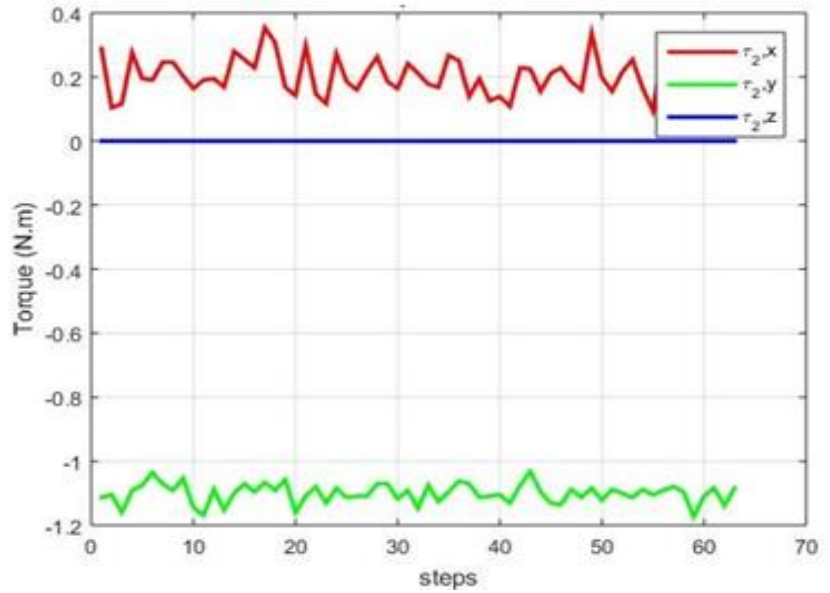

Fig. 10. Torque of body B

\section{DISCUSSION AND CONCLUSION}

In the present study, a wearable sensor system is designed and implemented which consists of two links, each link consists of three 3-axis accelerometers and one 9D0FIMU (3-axis accelerometer, 3-axis magnetometer and 3-axis gyroscope). This system is used to verify the new mathematical approaches for calculation of robot parameters. The tracking system presented in this research combines the well-established kinematic models designed for control of robots with state space methods to directly estimate the joint angles from wearable inertial sensors. The observation model is developed using Newton-Euler dynamics and kinematic chains $[18,19,20]$.

Velocity, acceleration and torque are recursively tracked and propagated from one link (limb) segment to another using Newton-Euler equations implemented in state 
space form. This study is composed of designing a circuit build, implementation, building mathematical model and verification phases. All these phases have been completed successfully. During the courses of the phases, the following conclusions have been made: Recently, the availability of low-cost wearable inertial sensors containing accelerometers, gyroscopes, and magnetometers has provided an alternative means to overcome the limitations of other motion capture systems.

Increasing the number and fusion of sensors leads to reducing or eliminating the drifts and errors. On the other hand, a fusion of magnetometers with the inertial sensors is useful in demonstrating enhanced performance when the magnetic field disturbances are absent. The primary challenge in manipulator and movement tracking and analysis is to design devices and algorithms that can accurately monitor movement regardless of the activity. Robot and $\mathrm{Hu}-$ man movement can be measured using a wide variety of techniques and sensors including optical, mechanical, magnetic, acoustic, or wearable inertial sensing systems. All these methods suffer from disadvantages. These systems are costly, can only be used in laboratory environments and hard to monitor daily activity.

The proposed sensor assembly overcomes these limitations. The case study showed accurate data capturing without any limitation. This paper has also demonstrated various formulas and algorithms that can help to calculate both robot parameters with these different sensors which help to find out angular acceleration, torque and angular velocity for smooth and reliable operations. The proposed circuitry system and algorithm showed very accurate results of these parameters.

\section{REFERENCES}

[1] V. Vikas and C. Crane, "Gyroscope-free link parameter measurement using accelerometers and magnetometer," in International Design Engineering Technical Conferences and Computers and Information, Buffalo, NY, 2014.

[2] V. Vikas and C. Crane, "Measurement of robot link joint parameters using multiple accelerometers and gyroscope," in International Design Engineering Technical Conferences and Computers and Information, Portland, OR, 2013. DOI: 10.1115/detc2013-12741

[3] P. Picerno, V. Viero, M. Donati, T. Triossi, V. Tancredi and G. Melchiorri, "Ambulatory assessment of shoulder abduction strength curve using a single wearable inertial sensor," Journal of Rehabilitation Research and Development, vol. 52, no. 2, pp. 171-182, 2015. DOI: ISSN: 2414-3103

\subsection{2/JRRD.2014.06.0146}

[4] R. E. Mayagoitia, V. Nene and P. H. Veltink, "Accelerometer and rate gyroscope measurement of kinematics: An inexpensive alternative to optical motion analysis systems," Journal of Biomechanics, vol. 35, no. 4, pp. 537-542, 2002.

DOI: $10.1016 / \mathrm{S} 0021-9290(01) 00231-7$

[5] A. T. M. Willemsen, J. A. Van Alste and H. B. K. Boom, “ Real-time gait assessment utilizing a new way of accelerometry," Journal of Biomechanics, vol. 23, no. 8, pp. 859-863, 1990.

DOI: $10.1016 / 0021-9290(90) 90033-Y$

[6] D. Gebre-Egziabher, G. H. Elkaim, J. D. Powell and B.

W. Parkinson, "A gyro-free quaternion-based at- titude determination system suitable for imple- mentation using low cost sensors," in Position Loca- $\quad$ tion and Navigation Symposium, San Diego, CA, 2000. DOI: $10.1109 /$ plans.2000.838301

[7] E. M. Foxlin, M. Harrington and Y. Altshuler, "Miniature six-DOF inertial system for tracking HMDs," in Aerospace/Defense Sensing and Con- $\quad$ trols, International Society for Optics and Photonics, $\quad$ Boston, MA, pp. 214-228, 1998.

[8] R. E. Mayagoitia, A. V. Nene and P. H. Veltink, "Ac- celerometer and rate gyroscope measurement of kinematics: An inexpensive alternative to opti- cal motion analysis systems," Journal of Biomechan- ics, vol. 35, no. 4, pp. 537-542, 2002.

DOI: $10.1016 / \mathrm{S} 0021-9290(01) 00231-7$

[9] E. Foxlin, " Pedestrian tracking with shoe-mounted inertial sensors. IEEE computer graphics and appli- cations," Computer Graphics and Applications, vol. 25,

no. 6, pp. 38-46,2005. DOI: $10.1109 /$ MCG. 2005.140

[10] D. Rotenberg, "Inertial and magnetic sensing of human motion," Ph.D. dissertation, University of Twente, Enschede, NA, 2006.

[11] P. Cheng and B. Oelmann, "Joint-angle measurement using accelerometers and gyroscopes a sur- $\quad$ vey," IEEE Transactions on Instrumentation and Mea- $\quad$ surement, vol. 59, no. 2, pp. 404-414, 2010.

DOI: 10.1109 /TIM.2009.2024367

[12] G. Baselli, G. Legnani, P. Franco, F. Brognoli, A. Mar- ras, F. Quaranta and B. Zappa, "Assessment of in- ertial and gravitational inputs to the vestibular sys- $\quad$ tem," Journal of Biomechanics, vol. 34, no. 6, pp. 821

- 826, 2001. DOI: 10.1016/S0021-9290(01)00012-4 
[13] G. Langfelder, C. Buffa, A. Frangi, A. Tocchio, E. Lasalandra and A. Longoni, "Z-axis magnetome- ters for MEMS inertial measurement units using an industrial process," IEEE Transactions on Industrial Electronics, vol. 60 , no. 9, pp. 3983-3990, $2013 . \quad$ DOI: 10.1109/TIE.2012.2210958

[14] K. Oberlander and N. Rer, "Inertial Measurement Unit (IMU) technology: Inverse kinematics: Joint considerations and the maths for deriving anatomical angles," 2016 [online]. Available: goo.gl/1gNQ98

[15] M. El-Gohary, "Joint angle tracking with inertial sen- $\quad$ sors" 2013 [online]. Available: goo.gl/XcBECV

[16] Z. Huiyu, H. Huosheng and T. Yaqin, "Inertial mea- $\quad$ surements of upper limb motion," Medical Bi-
479- 483, 2006. DOI: $10.1007 / \mathrm{s} 11517-006-0063-\mathrm{z}$

[17] J. J. Craig, Introduction to Robotics: Mechanics and Control. Reading, MA: Prentice Hall, 2005.

[18] T. Bajd, M. J. Mhelj Lenarcic, A. Stanovonic and M. Munih, Robotics. Dordrecht, NA: Springer, 2010.

[19] S. Morab and R. S. Pandey, "Propagation of Emic waves using Kappa distribution in the Jupiter's mag- $\quad$ netosphere," International Journal of Applied and Physical Sciences, vol. 3, no. 2, pp. 46-49, 2017.

[20] Y. Elhosane, A. Atway and Susianto, "Kinetic study of carbon dioxide absorption into glycine promoted methyl di ethanolamine (Mdea)," International Jour- nal of Technology and Engineering Studies, vol. 2, no. 2, pp. 47-52, 2016. DOI: 10.20469/ijtes.2.40003-2

ological Engineering \& Computing, vol. 44, no. 6, pp.

— This article does not have any appendix. — 